\title{
Canonical Wnt Signaling Promotes Macrophage Proliferation during Kidney Fibrosis
}

\author{
Ye Feng Yan Liang Jiafa Ren Chunsun Dai \\ Center for Kidney Disease, Second Affiliated Hospital of Nanjing Medical University, Nanjing, PR China
}

\author{
Keywords \\ Kidney · Fibrosis · Macrophage $\cdot$ Cell proliferation . \\ Wnt/ $\beta$-catenin
}

\begin{abstract}
Background: Wnt/ $\beta$-catenin, an evolutionary conserved signaling pathway, plays an essential role in modulating kidney injury and repair. Our previous studies demonstrated that Wnt/ $\beta$-catenin signaling could stimulate macrophage $M 2$ polarization and contribute to kidney fibrosis. However, whether canonical Wnt signaling activation leads to macrophage proliferation during kidney fibrosis remains to be determined. Methods: In this study, a mouse model with macrophage-specific $\beta$-catenin gene deletion was generated and a unilateral ureter obstruction (UUO) model was created. Results: In a mouse model with UUO nephropathy, deletion of $\beta$-catenin in macrophages attenuated macrophage proliferation and accumulation in kidney tissue. Wnt3a, a well-known canonical Wnt signaling stimulator, could markedly promote macrophage proliferation, whereas blocking canonical Wnt signaling with ICG-001 or ablating $\beta$-catenin could largely inhibit macrophage colony-stimulating factorstimulated macrophage proliferation. Wnt3a treatment could time-dependently upregulate cyclin D1 protein expression and blocking $\beta$-catenin signaling could downregu-
\end{abstract}

late it. Conclusion: These results demonstrate that Wnt/ $\beta$-catenin signaling is essential for promoting macrophage proliferation during kidney fibrosis. $\quad \odot 2018 \mathrm{~S}$. Karger AG, Basel

\section{Introduction}

Chronic kidney disease (CKD), defined as kidney damage and glomerular filtration rate $<60 \mathrm{~mL} / \mathrm{min} / 1.73 \mathrm{~m}^{2}$ for more than 3 months, affects more and more people around the world [1]. Regardless of the multitudinous causes for $\mathrm{CKD}$, patients suffering from it all come to one common pathway, namely renal destruction characterized by chronic inflammation and interstitial fibrosis [2]. Therefore, it is of great importance to decipher the cellular and molecular mechanisms of renal fibrosis and to identify an efficient therapeutic strategy for patients with CKD.

Chronic inflammatory reaction in kidney tissue is one of the major pathologic characteristics of CKD. Among all the inflammatory cell types accumulated in the fibrotic kidneys, macrophage is the pivotal one that participates in renal fibrosis $[2,3]$. It is well known that macrophage

\section{Y. Feng, Y. Liang, and J. Ren contributed equally to this paper.}

\section{KARGER}

(c) 2018 S. Karger AG, Basel

E-Mail karger@karger.com

www.karger.com/kdd
Chunsun Dai, MD, PhD

Center for Kidney Disease, Second Affiliated Hospital of Nanjing Medical University 262 North Zhongshan Road

Nanjing, Jiangsu 210003 (PR China)

E-Mail daichunsun@njmu.edu.cn 
accumulation and its phenotypic switch are two major decisive factors for renal fibrosis [4]. Macrophage proliferative expansion in situ leads to macrophage accumulation during kidney injury. Zhang et al. [5] reported that colony-stimulating factor 1 (CSF-1) is important for proliferative expansion of tissue macrophages. Blocking colony-stimulating factor receptor (c-fms) with anti-c-fms monoclonal antibody reduces macrophage proliferation in mice with unilateral ureter obstruction (UUO) or diabetic nephropathy $[6,7]$. Besides CSF-1, IL-4 is also able to drive the proliferation of tissue macrophages [8-10]. As macrophage expansion is crucial for kidney fibrosis, the other mechanisms regulating macrophage proliferation during kidney fibrosis need to be further explored.

Wnt signaling is categorized into the canonical and noncanonical signaling pathways based on whether $\beta$-catenin is activated or not. The canonical Wnt signaling cascade controls many biological events such as cell differentiation, proliferation, and polarization, all of which are involved in kidney injury and repair $[11,12]$. In adult kidneys, Wnt/ $\beta$-catenin signaling is relatively silenced. However, upon occurrence of renal injury, aberrant activation of Wnt/ $\beta$-catenin signaling can be observed. It has been reported that Wnt ligands secreted into the extracellular environment bind to the Frizzled/LRP receptors on the cell membrane, thereafter stabilize $\beta$-catenin in the cytoplasm, accumulate $\beta$-catenin in the cytoplasm, then translocate into the nucleus and bind TCF/LEF, which stimulates the transcription of many target genes, such as cyclin D1 and c-myc, and drive cell proliferation $[13,14]$. Our previous study showed that during kidney fibrosis, Wnt/ $\beta$-catenin activation may drive macrophage alternative activation and contribute to kidney fibrosis [15]. However, whether canonical Wnt signaling activation leads to macrophage proliferation during kidney fibrosis remains to be determined.

In this study, we report that $\mathrm{Wnt} / \beta$-catenin signaling activation can promote macrophage proliferation. Blocking canonical Wnt signaling downregulates cyclin D1 expression and attenuates cell proliferation in cultured macrophages. In mice with UUO nephropathy, deletion of $\beta$-catenin in macrophages diminishes macrophage proliferation and accumulation, which is accompanied by less kidney fibrosis.

\section{Materials and Methods}

Cell Culture and Treatment

Raw 264.7 cells were cultured in Dulbecco's modified Eagle's medium (DMEM) containing 10\% (v/v) fetal bovine serum (FBS) (Invitrogen, Grand Island, NY, USA) and 1\% (v/v) antibiotics
(100 U/mL penicillin) at $37^{\circ} \mathrm{C}$ in $5 \% \mathrm{CO}_{2}$. Bone marrow-derived macrophages (BMMs) were isolated from the bone marrow as previously described [16]. BMMs were cultured in DMEM containing $10 \%(\mathrm{v} / \mathrm{v}) \mathrm{FBS}, 10 \mathrm{ng} / \mathrm{mL}$ of mouse macrophage colony-stimulating factor (M-CSF) (cat: 416-ML-050, R\&D), and 1\% (v/v) antibiotics for 9 days. The medium was changed every other day. To generate BMMs with $\beta$-catenin gene deletion, BMMs isolated from Csf1r-Cre+/-, $\beta$-catenin ${ }^{\mathrm{fL} / \mathrm{fL}}$ mice were treated with $1 \mu \mathrm{M} 4$-hydroxytamoxifen (4-OHT) (H6278, Sigma-Aldrich) at the beginning of the culture. BMMs isolated from Csf1r-Cre-/-, $\beta$-cate$\operatorname{nin}^{\mathrm{fLl} / \mathrm{fLl}}$ mice were treated with $4-\mathrm{OHT}$ as control [15].

\section{Mice and Animal Models}

Male C57BL/6 mice weighing approximately 18-20 g were acquired from the specific Pathogen-Free Laboratory Animal Center of Nanjing Medical University. Homozygous $\beta$-catenin floxed mice $(022775, \mathrm{~B} 6[\mathrm{Cg}]-\mathrm{Ctnnb} 1 \mathrm{tm} 1 \mathrm{Knw} / \mathrm{J})$ and mice expressing tamoxifen-inducible MerCreMer fusion protein under the control of macrophage-specific mouse Csf1r promoter (019098, FVBTg[Csf1r-cre/Esr1*]) were ordered from Jackson Laboratories (Bar Harbor, ME, USA) $[15,17]$. FVB-Tg(Csf1r-cre/Esr1*) mice were crossed with $\mathrm{C} 57 \mathrm{BL} / 6 \mathrm{~J}$ mice for eight generations to get Csf1r-Cre transgenic mice on a C57BL/6J background. All animals were housed in the specific pathogen-free laboratory animal center of Nanjing Medical University according to the guidelines of the Institutional Animal Care and Use Committee of Nanjing Medical University, Nanjing, China. The generation of the mice with genotyping Csf1r-Cre+/-, $\beta$-catenin ${ }^{\mathrm{fL} / \mathrm{fL}}$ and the same gender littermates with genotyping Csf1r-Cre-/-, $\beta$-catenin ${ }^{\mathrm{fL} / \mathrm{fL}}$ were performed. Csf1r-Cre $+/-, \beta$-catenin ${ }^{\mathrm{fL} / \mathrm{fL}}$ mice and control littermates were intraperitoneally injected with tamoxifen (T5648, Sigma-Aldrich) at $25 \mathrm{mg} / \mathrm{kg}$ for 5 consecutive days, and 2 days after the last injection the mice were subjected to UUO operation as previously reported $[15,18]$.

\section{Western Blot Analysis}

Raw 264.7 cells and BMMs were lysed in $1 \times$ SDS sample buffer. The kidneys were lysed with RIPA buffer containing $1 \%$ NP-40, $0.1 \%$ SDS, $100 \mathrm{mg} / \mathrm{mL}$ of PMSF, $1 \%$ protease inhibitor mixture, and $1 \%$ phosphatase I and II inhibitor mixture on ice. The supernatants were collected after centrifugation at $13,000 \mathrm{~g}$ at $4{ }^{\circ} \mathrm{C}$ for $30 \mathrm{~min}$. The primary antibodies used were anti-cyclin D1 (cat: RB9041-P0; Thermo) and anti-PCNA (cat: sc9857; Santa Cruz Biotechnology). Quantification was performed by measuring the intensity of the signals with the aid of the National Institutes of Health Image software package.

\section{Immunofluorescent Staining}

Kidney cryosections at $3 \mu \mathrm{m}$ thickness were fixed for $15 \mathrm{~min}$ with $4 \%$ paraformaldehyde, followed by permeabilization with $0.2 \%$ Triton $\mathrm{X}-100$ in $1 \times \mathrm{PBS}$ for $5 \mathrm{~min}$ at room temperature. After blocking with $2 \%$ donkey serum for $60 \mathrm{~min}$, slides were immunestained with the antibodies anti-F4/80 (cat: 14-4801; eBioscience, San Diego, CA, USA) and anti-Ki67 (cat: ab15580; Abcam, Cambridge, UK). Raw 264.7 cells or BMMs cultured on coverslips were washed with cold $1 \times$ PBS and fixed with cold methanol/acetone (1:1) for $10 \mathrm{~min}$ at $-20^{\circ} \mathrm{C}$. After three extensive washings with $1 \times$ PBS, cells were treated with $0.1 \%$ Triton X-100 for 5 min and blocked with $2 \%$ normal donkey serum in $1 \times$ PBS buffer for $40 \mathrm{~min}$ at room temperature and incubated with anti-Ki67 (cat: ab15580; 


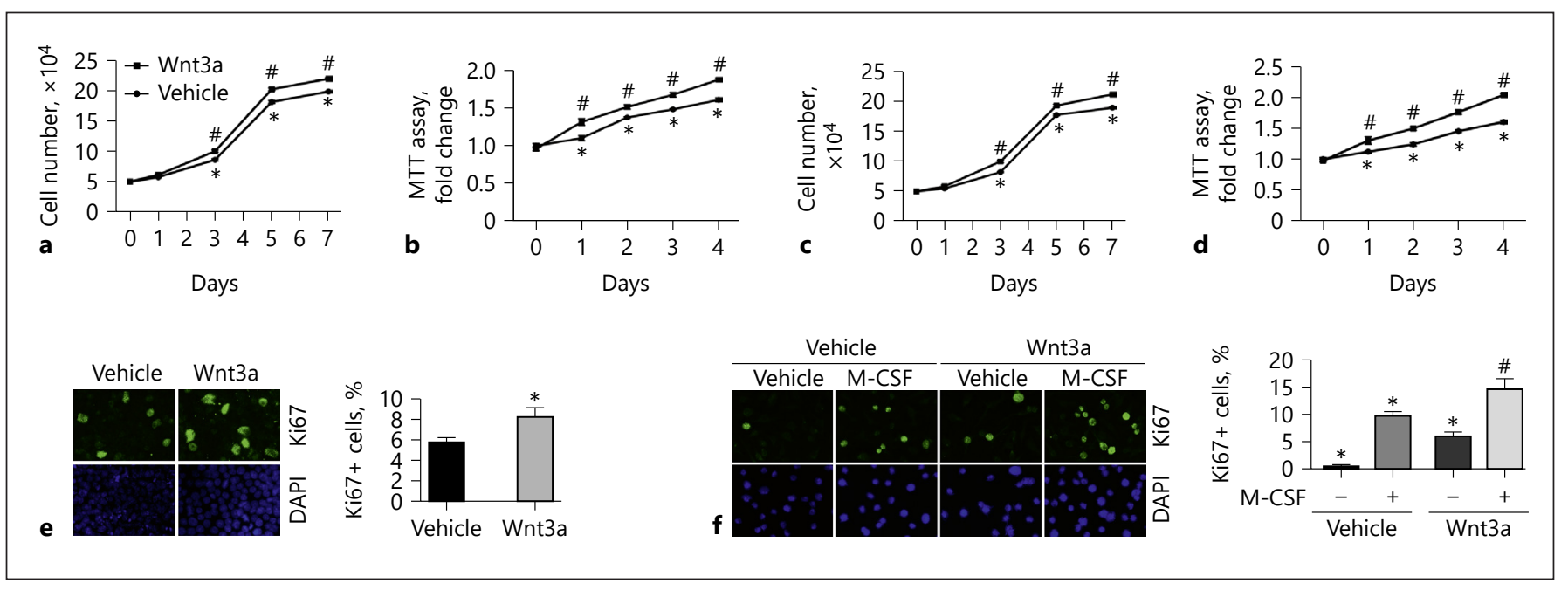

Fig. 1. Wnt3a stimulates macrophage proliferation. a, b Cell counting (a) and MTT assay (b) in Raw 264.7 cells. ${ }^{*} p<0.05$ versus cells at day $0, n=5 ;{ }^{\#} p<0.05$ versus cells without Wnt 3 a treatment at the respective time, $n=5$. c, $\mathbf{d}$ Cell counting (c) and MTT assay (d) in BMMs. ${ }^{*} p<0.05$ versus cells at day $0, n=5 ;{ }^{*} p<0.05$ versus cells without Wnt3a treatment at the respective time, $n=5$. e Representative immunofluorescent staining images (left) and quantitative analysis (right) for Ki67 in Raw 264.7 cells. ${ }^{*} p<0.05$ versus cells treated with vehicle alone, $n=4$. Cells were stained with DAPI to visualize the nuclei. f Representative immunofluorescent images (left) and quantitative analysis (right) for Ki67 in BMMs. ${ }^{*} p<0.05$ versus cells treated with vehicle alone, $n=4$; $\# p<0.05$ versus cells stimulated with M-CSF for $24 \mathrm{~h}, n=4$. BMMs, bone marrow-derived macrophages; DAPI, 4',6-diamidino2-phenylindole; M-CSF, macrophage colony-stimulating factor; MTT, 3-(4,5-dimethylthiazol-2-yl)-2-5-diphenyltetrazolium bromide.
Abcam), followed by staining with fluorescein isothiocyanate or tetramethylrhodamine-conjugated secondary antibodies. Cells were also stained with $4^{\prime}$,6-diamidino-2-phenylindole to visualize the nuclei. Slides were viewed with a Nikon Eclipse 80i Epi-fluorescence microscope equipped with a digital camera. The F4/80and Ki67-positive macrophage number was counted from ten randomly selected fields in the cortical area for each sample under a $400 \times$ microscope and an average number of positive cells for each section was calculated.

\section{Macrophage Proliferation Assay}

For cell counting, Raw 264.7 cells or BMMs were seeded in 24well plates at $5 \times 10^{4}$ cells per well and cultured with DMEM containing 10\% FBS and M-CSF. The culture medium was changed every day. Cells were counted on days $1,3,5$, and 7 after plating. For the 3-(4,5-dimethylthiazol-2-yl)-2-5-diphenyltetrazolium bromide (MTT) assay, macrophages were seeded in 96-well plates at $3 \times 10^{4}$ cells per well and cultured with complete DMEM containing M-CSF. The culture medium was changed every day. Ten microliters of MTT reagent $(5 \mathrm{mg} / \mathrm{mL}$ ) (Sigma Chemical Co., St. Louis, MO, USA) per well was added and incubated for $4 \mathrm{~h}$ at $37^{\circ} \mathrm{C}$. The culture medium was taken out and cells were washed with cold $1 \times$ PBS three times. One hundred microliters of dimethyl sulfoxide was added to lyse the cells and solubilize colored crystals, and optical density was read at $560 \mathrm{~nm}$ wavelength.

\section{Flow Cytometry}

Single-cell suspensions were prepared from kidneys as described previously [15] and incubated with anti-CD45-FITC
(103107; Biolegend), anti-CD11b-APC (101220; Biolegend), and anti-Ki67-PE (652403; Biolegend). Fixation/permeabilization diluent (00-5223-56; eBioscience) was used for fixation and permeabilization according to the manufacturer's instruction. For cell cycle analysis, the Cell Cycle Assay Kit (A411-01/02; Vazyme) was used according to the manufacturer's instruction. Cells were acquired on a BD Canto II Flow Cytometer with the FlowJo software.

\section{Statistical Analyses}

All data examined are presented as mean \pm SEM. Statistical analysis of the data was performed using the SigmaStat software (Jandel Scientific Software, San Rafael, CA, USA). Comparison between groups was made using one-way ANOVA followed by the Student-Newman-Keuls test. For cell counting and MTT assay, comparison between groups was made using two-way ANOVA followed by the Student-Newman-Keuls test. The Student paired or unpaired $t$ test was used to compare two groups. A $p$ value $\leq 0.05$ was considered statistically significant.

\section{Results}

\section{Wnt3a Promotes Macrophage Proliferation}

To investigate the role of $\mathrm{Wnt} / \beta$-catenin in regulating macrophage proliferation, we treated Raw 264.7 cells, a mouse monocyte/macrophage cell line, with Wnt3a for 7 days. As shown in Figure 1a, Wnt3a (100 ng/mL) largely 


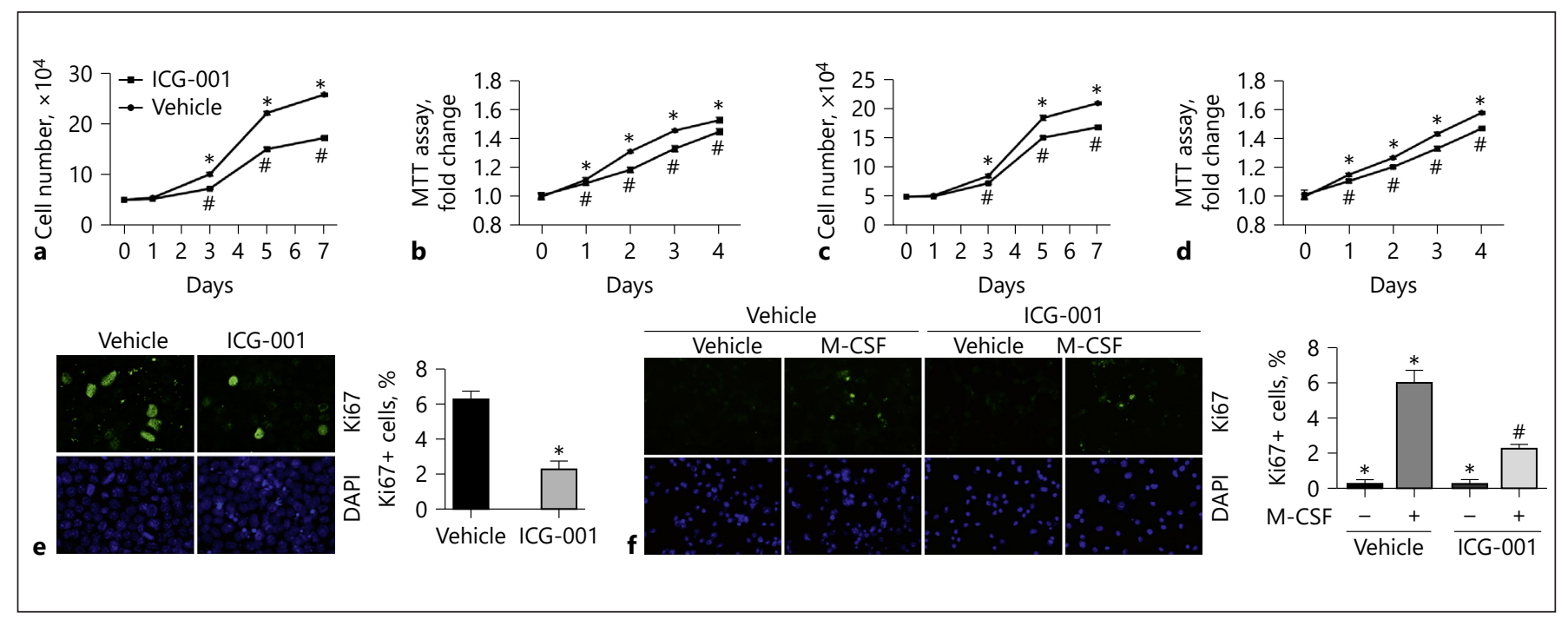

Fig. 2. Blockade of $\beta$-catenin signaling reduces macrophage proliferation. a, b Cell counting (a) and MTT assay (b) in Raw 264.7 cells. ${ }^{*} p<0.05$ versus cells at day $0, n=5$; ${ }^{\#} p<0.05$ versus cells without ICG-001 treatment at the respective time, $n=5$. c, d Cell counting (c) and MTT assay (d) in BMMs. ${ }^{*} p<0.05$ versus cells at day $0, n=5 ;{ }^{*} p<0.05$ versus cells without ICG-001 treatment at the respective time, $n=5$. e Representative immunofluorescent images (left) and quantitative analysis (right) for Ki67 in Raw 264.7 cells. ${ }^{*} p<0.05$ versus control, $n=4$. Cells were stained with DAPI to visualize the nuclei. f Representative immunofluorescent images (left) and quantitative analysis (right) for Ki67 in BMMs. ${ }^{*} p<0.05$ versus cells treated with vehicle alone, $n=4$; ${ }^{*} p<0.05$ versus cells stimulated with M-CSF, $n=4$. Cells were stained with DAPI to visualize the nuclei. BMMs, bone marrow-derived macrophages; DAPI, 4' , 6-diamidino-2-phenylindole; M-CSF, macrophage colony-stimulating factor; MTT, 3-(4,5-dimethylthiazol2-yl)-2-5-diphenyltetrazolium bromide. increased the cell number from day 3 to 7 in Raw 264.7 cells compared to those treated with vehicle. MTT assay revealed similar results (Fig. 1b). In BMMs, Wnt3a (100 $\mathrm{ng} / \mathrm{mL}$ ) markedly stimulated BMM proliferation compared to those treated with M-CSF alone (Fig. 1c, d). In addition, Wnt3a also significantly upregulated Ki67 expression in both Raw 264.7 cells and BMMs (Fig. 1e, f). Together, these results demonstrated that $\mathrm{Wnt} / \beta$-catenin activation is able to stimulate macrophage proliferation.

\section{Blockade of $\beta$-Catenin Signaling Reduces Macrophage Proliferation}

The data above demonstrated that Wnt/ $\beta$-catenin activation can promote macrophage proliferation. To further investigate whether $\beta$-catenin activation is required for macrophage proliferation, we first treated Raw 264.7 cells and BMMs with ICG-001, a small molecule that specifically inhibits $\mathrm{T}$-cell factor/ $\beta$-catenin transcription in a cyclic AMP response-element binding protein (CBP)-dependent fashion, to block $\beta$-catenin signaling. Raw 264.7 cells were starved with serum-free medium overnight, then cultured with DMEM containing 10\% FBS to stimulate cell proliferation. Macrophage proliferation was markedly increased from day 3 to 7 after stimulation, whereas ICG-001 $(10 \mu \mathrm{g} / \mathrm{mL})$ markedly inhibited it (Fig. 2a, b). BMM proliferation was markedly stimulated by M-CSF from days 3, 5, and 7 after plating, and ICG$001(10 \mu \mathrm{g} / \mathrm{mL})$ largely decreased M-CSF-stimulated macrophage proliferation (Fig. 2c, d). Furthermore, immune staining of Ki67 showed that ICG-001 largely decreased FBS and M-CSF-induced Ki67 protein expression in Raw 264.7 cells and BMMs, respectively (Fig. 2e, f).

We also created primary cultured mouse BMMs with tamoxifen-inducible $\beta$-catenin deletion. BMMs were isolated from Csf1r-Cre+- $\beta$-catenin ${ }^{\mathrm{fL} / \mathrm{fL}}$ and Csf1r-Cre- $\beta$ catenin ${ }^{\mathrm{fL} / \mathrm{fL}}$ mice, respectively. Beta-catenin gene deletion was induced in BMMs from Csf1r-Cre+- $\beta$-catenin ${ }^{\mathrm{fL} / \mathrm{fL}}$ mice after 4-OHT treatment as shown in our published study [15]. BMM proliferation was stimulated at days 3, 5 , and 7 after M-CSF treatment in $\beta$-catenin $+/+$ BMMs, whereas deletion of $\beta$-catenin remarkably diminished cell proliferation (Fig. 3a, b). Immune staining showed that the number of macrophages staining positive for Ki67 was also much less in $\beta$-catenin-/- BMMs compared to those in $\beta$-catenin $+/+$ BMMs (Fig. 3c). FACS analysis 


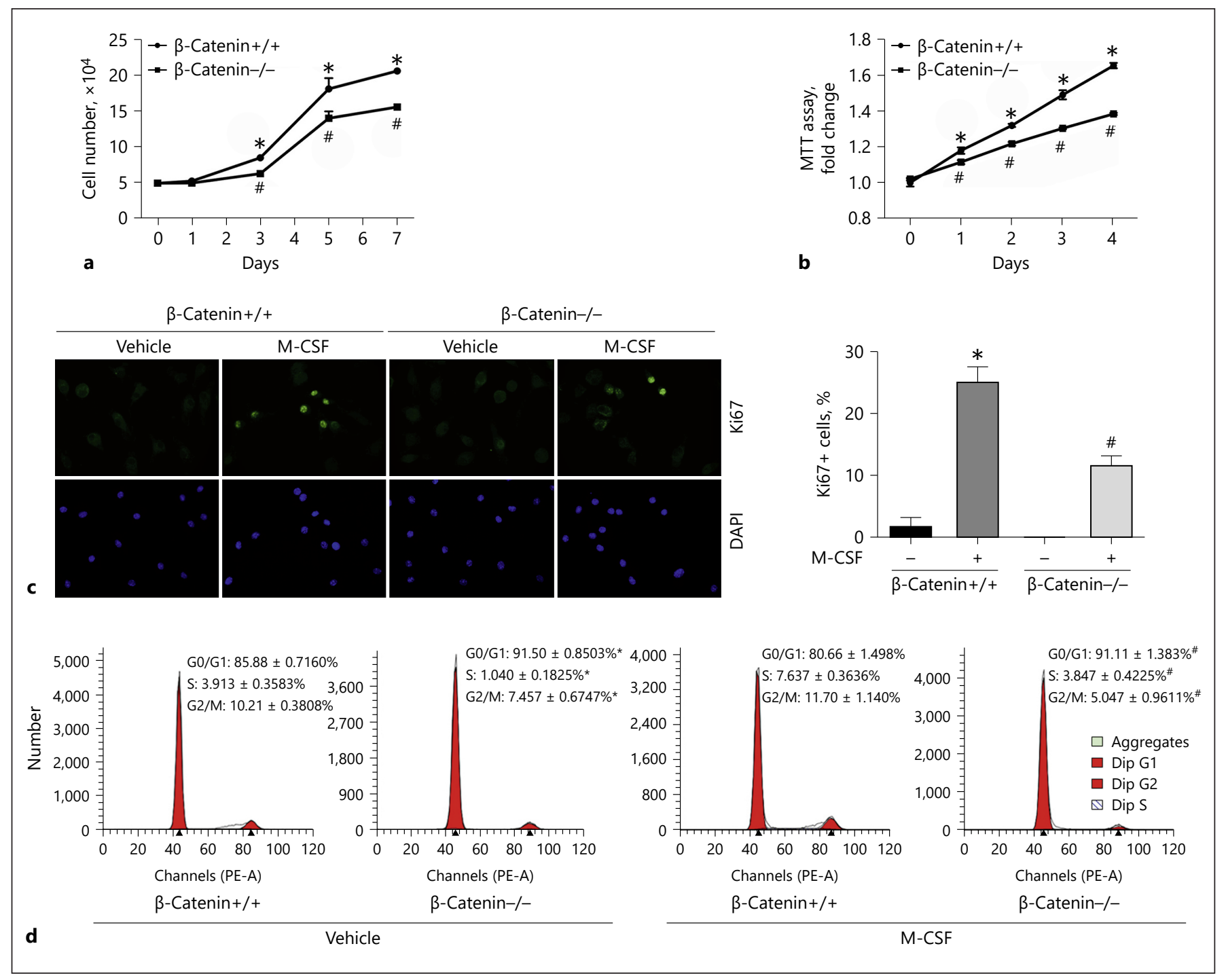

Fig. 3. Ablation of $\beta$-catenin in macrophage reduces macrophage proliferation. a, b Cell counting (a) and MTT assay (b) in $\beta$ catenin $+/+$ or $\beta$-catenin-/-BMMs. ${ }^{*} p<0.05$ versus $\beta$-catenin $+/+$ BMMs at day $0, n=5 ;{ }^{\#} p<0.05$ versus $\beta$-catenin $+/+$ BMMs at the respective time, $n=5$. c Representative immunofluorescent staining images (left) and quantitative analysis (right) for Ki67 in $\beta$-catenin $+/+$ and $\beta$-catenin-/- BMMs. ${ }^{*} p<0.05$ versus $\beta$-catenin $+/+$ BMMs treated with vehicle alone, $n=3 ;{ }^{\#} p<0.05$ versus $\beta$-catenin $+/+$ BMMs treated with M-CSF, $n=3$. Cells were stained

showed that the percentage of G0/G1 cells in $\beta$-catenin-/BMMs was much higher than that in $\beta$-catenin $+/+$ BMMs, indicating that deletion of $\beta$-catenin prevents cells from entering the cell cycle by stopping them in the G0/G1 phase (Fig. 3d). Thus, it may be concluded that $\beta$-catenin signaling activation is both sufficient and required for promoting macrophage proliferation.

Beta-Catenin Promotes Macrophage Proliferation with DAPI to visualize the nuclei. d FACS analysis showing cell cycle for $\beta$-catenin $+/+$ and $\beta$-catenin-/-BMMs. ${ }^{*} p<0.05$ versus $\beta$-catenin $+/+$ BMMs treated with vehicle alone, $n=3$; ${ }^{\#} p<0.05$ versus $\beta$-catenin $+/+$ BMMs treated with $M-C S F(10 \mathrm{ng} / \mathrm{mL})$, $n=3$. BMMs, bone marrow-derived macrophages; DAPI, $4^{\prime}, 6-\mathrm{di}-$ amidino-2-phenylindole; M-CSF, macrophage colony-stimulating factor; MTT, 3-(4,5-dimethylthiazol-2-yl)-2-5-diphenyltetrazolium bromide.

\section{Beta-Catenin Signaling Regulates the Expression of Cyclin D1 in Macrophages}

Cyclin D1, one of the critical genes involved in cell proliferation, is the target gene of $\mathrm{Wnt} / \beta$-catenin signaling $[14,19]$. In this study, we found that Wnt3a upregulated cyclin D1 protein expression in both Raw 264.7 cells and BMMs in a time-dependent manner (Fig. 4a, b). Sim- 


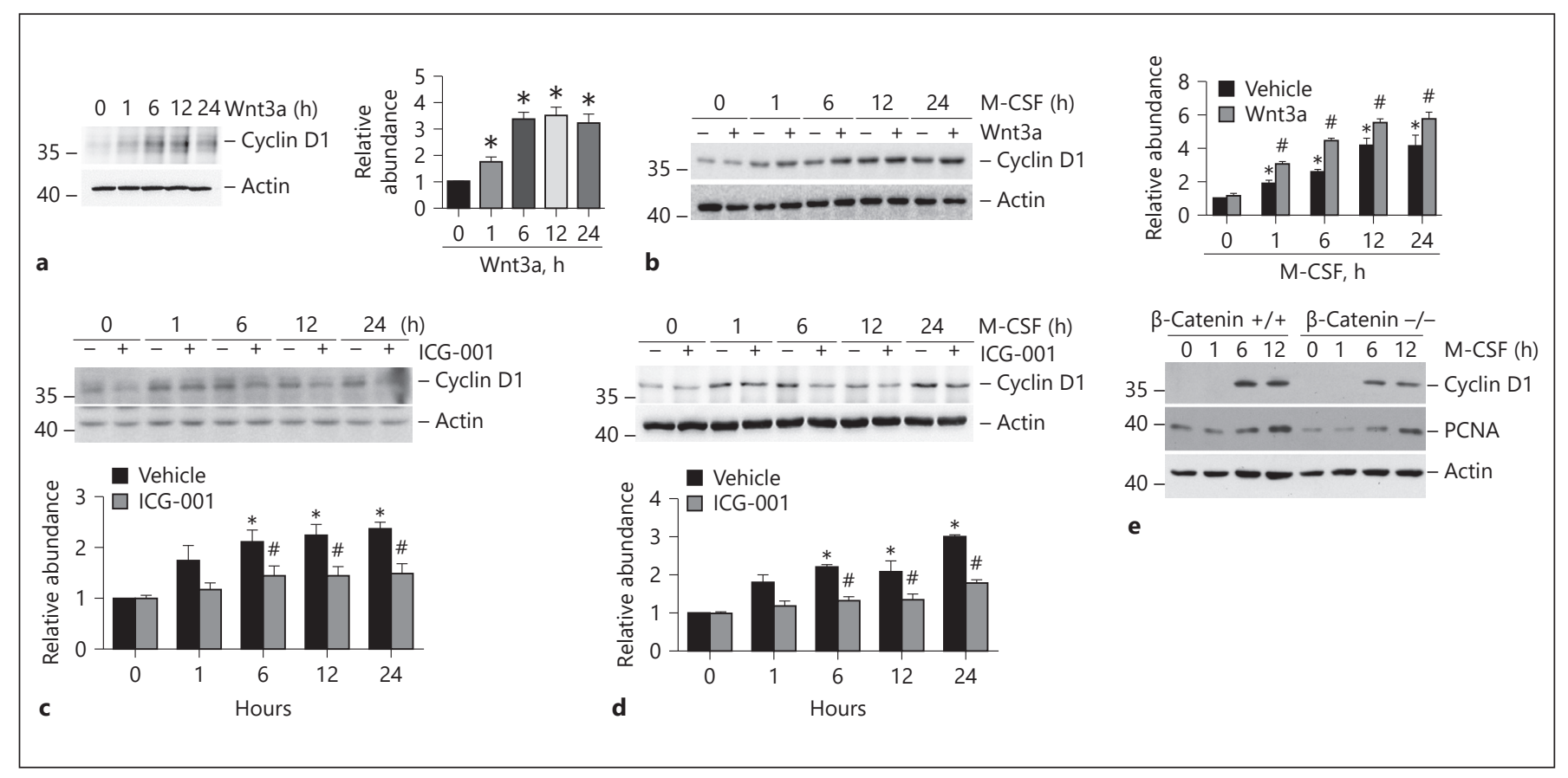

Fig. 4. Regulation of $\beta$-catenin signaling in the expression of cyclin D1 in macrophages. a Western blotting analyses (left) and quantitative analysis (right) showing the induction of cyclin D1 in Raw 264.7 cells treated with Wnt3a $(100 \mathrm{ng} / \mathrm{mL}) .{ }^{*} p<0.05$ versus cells treated with vehicle alone, $n=3$. $\mathbf{b}$ Western blotting analyses (left) and quantitative analysis (right) showing the induction of cyclin D1 protein after $\mathrm{Wnt} 3 \mathrm{a}(100 \mathrm{ng} / \mathrm{mL})$ treatment in the presence of M-CSF in BMMs. ${ }^{*} p<0.05$ versus cells treated with vehicle alone, $n=3$; ${ }^{\#} p<0.05$ versus cells treated with M-CSF alone at the respective time, $n=3$. c Western blotting analyses (upper) and quantitative analysis (lower) showing the reduction of cyclin D1 protein after ICG-001 treatment in Raw 264.7 cells. ${ }^{*} p<0.05$ versus cells

ilarly, Western blotting assay and quantitative analyses showed that cyclin D1 protein abundance was induced at $1,6,12$, and $24 \mathrm{~h}$ in both Raw 264.7 cells (Fig. 4c) and BMMs (Fig. 4d) after stimulation with 10\% FBS and M-CSF, respectively. ICG-001 significantly inhibited cyclin D1 expression at 6,12 , and $24 \mathrm{~h}$ after stimulation in both cell types. In addition, Western blot analyses revealed that cyclin D1 and PCNA protein expression was induced at 6 and $12 \mathrm{~h}$ after M-CSF treatment in $\beta$-catenin $+/+$ BMMs, while $\beta$-catenin deletion largely downregulated M-CSF-stimulated cyclin D1 and PCNA expression (Fig. 4e).

\section{Ablation of $\beta$-Catenin in Macrophages Diminishes \\ Macrophage Proliferation in UUO Kidneys}

Macrophage accumulation within the fibrotic kidneys is determined by macrophage infiltration, proliferation, treated with vehicle alone, $n=3 ;{ }^{*} p<0.05$ versus cells without ICG001 treatment at the respective time, $n=3$. d Western blotting analyses (upper) and quantitative analysis (lower) showing the reduction of cyclin D1 protein after ICG-001 treatment in BMMs in the presence of M-CSF. ${ }^{*} p<0.05$ versus cells treated with vehicle alone, $n=3$; ${ }^{*} p<0.05$ versus cells treated with $\mathrm{M}$-CSF alone at the respective time, $n=3$. e Western blotting analyses showing the reduction of cyclin D1 and PCNA protein in $\beta$-catenin-/- BMMs compared to $\beta$-catenin $+/+$ BMMs after M-CSF stimulation. $\mathrm{BMMs}$, bone marrow-derived macrophages; M-CSF, macrophage colony-stimulating factor.

and cell death [20]. Our previous study showed that deletion of $\beta$-catenin in macrophages attenuated UUO nephropathy in mice [15]. In order to further clarify the role of $\beta$-catenin in regulating macrophage proliferation during kidney fibrosis, we first performed co-immune staining with Ki67 and F4/80 to identify macrophage proliferation in the kidneys. Few F4/80 and Ki67 costaining positive cells were found in sham kidneys. At day 14 after UUO, about $2.7 \%$ of the F4/80-positive cells expressed Ki67 in Mac- $\beta$-catenin+/+ kidneys, which was less in the knockout kidneys (Fig. 5a, b). Furthermore, we examined macrophage proliferation by flow cytometric assay. As shown in Figure 5c, CD11b+/Ki67+ cells were much less in the knockout kidneys after UUO compared to those from control littermates. Together, these data suggest that ablation of $\beta$-catenin in macrophages attenuates macrophage proliferation in UUO kidneys. 


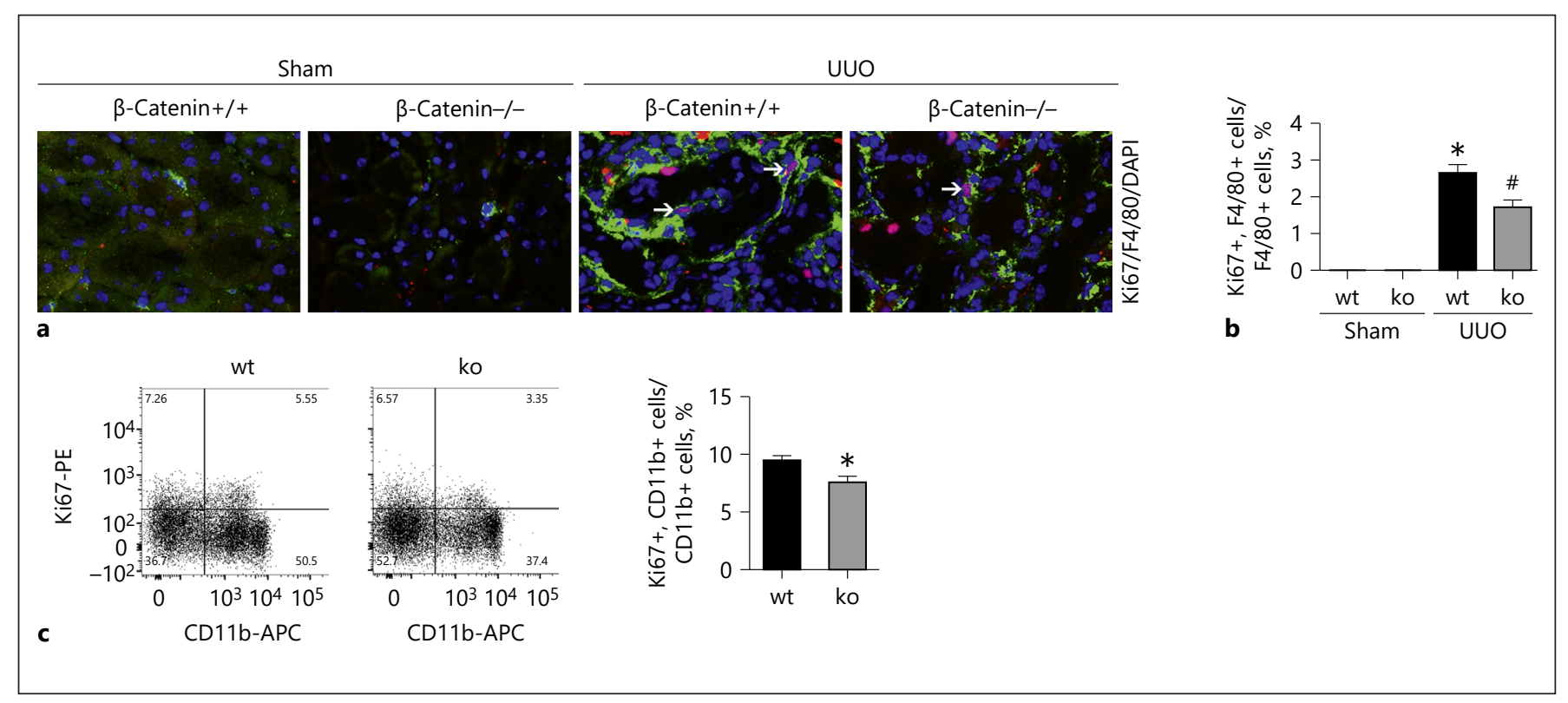

Fig. 5. Ablation of $\beta$-catenin in macrophages attenuates macrophage proliferation in the kidneys with UUO nephropathy. a, b Representative images for costaining of F4/80 and Ki67 (a) and quantitative analysis for Ki67- and F4/80-positive macrophages in kidney tissues (b). Arrows indicate costaining-positive macrophages. ${ }^{*} p<0.05$ versus $\beta$-catenin $+/+$ sham control, $n=5$; ${ }^{\#} p<$
0.05 versus $\beta$-catenin $+/+$ control littermates after UUO, $n=5$. c FACS analysis showing macrophage proliferation for macrophages from UUO kidneys. ${ }^{*} p<0.05$ versus macrophages from Mac- $\beta$-catenin+/+ UUO kidneys, $n=4$. UUO, unilateral ureter obstruction.

\section{Discussion}

We report here that $\mathrm{Wnt} / \beta$-catenin signaling activation is essential for promoting macrophage proliferation. In UUO kidneys, targeting $\beta$-catenin leads to less macrophage proliferation and kidney fibrosis. Thus, it may be concluded that $\mathrm{Wnt} / \beta$-catenin-promoted macrophage proliferation contributes to macrophage accumulation and kidney fibrosis in mice with UUO nephropathy.

Wnt signaling can be divided into canonical $(\beta$-catenindependent) and noncanonical ( $\beta$-catenin-independent) pathways [21]. Canonical Wnt signaling is essential for embryogenesis and organogenesis. In the fetal kidneys, $\mathrm{Wnt} / \beta$-catenin drives branching nephrogenesis [22]. Although the Wnt pathway is important in embryonic development, aberrant activation of the Wnt pathway is associated with the pathogenesis of many kinds of disease [23]. Familial adenomatous polyposis, in which aberrant $\mathrm{Wnt} / \beta$-catenin signaling activation leads to increased cell proliferation and adenomatous lesions, is the best-known example [24]. In addition, aberrant $\mathrm{Wnt} / \beta$-catenin signaling activation also participates in the carcinogenesis of colorectal and liver cancer [12, 25]. Recently, an increas- ing number of evidence revealed that sustained Wnt/ $\beta$-catenin signaling activation is related to fibrotic disease in many types of tissue and organ [26-29]. Wnt3a, a canonical Wnt pathway stimulator, is reported to induce lung fibroblast migration and proliferation, thus contributing to pulmonary fibrosis [26]. In addition to the fibrotic disease mentioned above, many experiments using gain- and loss-of-function of Wnt signaling clearly demonstrated that hyperactive $\mathrm{Wnt} / \beta$-catenin signaling is pivotal for promoting the progression of CKD [30]. In animal models with CKD, most of the Wnt proteins are upregulated. Upregulated Wnt proteins may trigger podocyte injury or interstitial fibrosis in mice with Adriamycin nephropathy or UUO nephropathy [30, 31]. In addition to animal models, the relationship of Wnt/ $\beta$-catenin signaling activation with CKD has also been reported in patients with IgA nephropathy, HIV-induced nephropathy, diabetic kidney disease, and focal segmental glomerulosclerosis [32, 33]. A microarray analysis study revealed that the expression profile of a subset of $21 \mathrm{Wnt}$-related genes may differentiate patients with IgA nephropathy from healthy controls. It is of note that the upregulation of Wnt genes is associated with enhanced peripheral 
blood mononuclear cell proliferation and activation in patients with IgA nephropathy [34].

Macrophages participate in interstitial fibrosis and hence contribute to the initiation and progression of CKD. Sustained accumulation and activation of macrophages in kidney tissue may result in the production of multiple profibrotic cytokines and kidney fibrosis [35]. It has been reported that blockage of Wnt secretion by a small-molecule inhibitor of porcupine C59 or treatment with ICG-001 can ameliorate UUO nephropathy in mice $[36,37]$. Our previous study reported that $\mathrm{Wnt} / \beta$-catenin signaling activation in macrophages promotes macrophage accumulation and leads to kidney fibrosis in a mouse model with UUO nephropathy. In addition, Wnt/ $\beta$-catenin signaling can stimulate macrophage M2 polarization [15]. It is well known that the accumulation of macrophages in the diseased kidneys may result from the infiltration of circulating monocytes and proliferation of macrophages in situ $[38,39]$. In this study, we found that Wnt3a markedly stimulated cyclin D1 protein expression and cell proliferation in cultured macrophages. Cyclin D1 is one of the target genes of canonical Wnt signaling, which drives cell proliferation in many cell types [40,41]. Therefore, it may be concluded that the induction of cyclin D1 mediates $\beta$-catenin-promoted macrophage proliferation. In mice with macrophage $\beta$-catenin gene deletion, macrophage accumulation and proliferation in UUO kidneys were less compared to those in control littermates, which further demonstrated that $\beta$-catenin is essential for driving macrophage proliferation during kidney fibrosis.
Together, in addition to promoting macrophage M2 polarization as we previously reported, this study showed that $\mathrm{Wnt} / \beta$-catenin signaling activation can promote macrophage proliferation and macrophage accumulation, which may participate in kidney fibrosis in mice with UUO nephropathy.

\section{Acknowledgment}

This work was supported by National Science Foundation of China grants 81570611/H0503 and 81770675/H0503 and Science Foundation of Jiangsu Province grant BK20140048 to C. Dai.

\section{Statement of Ethics}

The animal protocols used in this study were approved by the Institutional Animal Care and Use Committee of Jiangsu Province (Jiangsu Government Publication No. 45, revised, 2008).

\section{Disclosure Statement}

The authors declare that no competing interest exists.

\section{Author Contributions}

Y. Feng, Y. Liang, and J. Ren performed the experiments and wrote the manuscript. C. Dai supervised the work and revised the manuscript.

\section{References}

1 Webster AC, Nagler EV, Morton RL, Masson P: Chronic kidney disease. Lancet 2017;389: 1238-1252.

2 Eddy AA: Progression in chronic kidney disease. Adv Chronic Kidney Dis 2005;12:353365.

3 Vernon MA, Mylonas KJ, Hughes J: Macrophages and renal fibrosis. Semin Nephrol 2010;30:302-317.

4 Liu Y: Cellular and molecular mechanisms of renal fibrosis. Nat Rev Nephrol 2011;7:684696.

5 Zhang MZ, Yao B, Yang S, Jiang L, Wang S, Fan X, et al: CSF-1 signaling mediates recovery from acute kidney injury. J Clin Invest 2012;122:4519-4532.

6 Lim AK, Ma FY, Nikolic-Paterson DJ, Thomas MC, Hurst LA, Tesch GH: Antibody blockade of $\mathrm{c}$-fms suppresses the progression of inflammation and injury in early diabetic ne- phropathy in obese $\mathrm{db} / \mathrm{db}$ mice. Diabetologia 2009;52:1669-1679.

-7 Le Meur Y, Tesch GH, Hill PA, Mu W, Foti R, Nikolic-Paterson DJ, et al: Macrophage accumulation at a site of renal inflammation is dependent on the M-CSF/c-fms pathway. J Leukoc Biol 2002;72:530-537.

8 MacDonald AS, Loke P, Martynoga R, Dransfield I, Allen JE: Cytokine-dependent inflammatory cell recruitment patterns in the peritoneal cavity of mice exposed to the parasitic nematode Brugia malayi. Med Microbiol Immunol 2003;192:33-40.

-9 Anthony RM, Urban JF Jr, Alem F, Hamed HA, Rozo CT, Boucher JL, et al: Memory T(H)2 cells induce alternatively activated macrophages to mediate protection against nematode parasites. Nat Med 2006;12:955-960.

10 Jenkins SJ, Ruckerl D, Cook PC, Jones LH, Finkelman FD, van Rooijen N, et al: Local macrophage proliferation, rather than recruitment from the blood, is a signature of TH2 inflammation. Science 2011;332:12841288.

11 DiRenzo DM, Chaudhary MA, Shi X, Franco SR, Zent J, Wang K, et al: A crosstalk between TGF- $\beta /$ Smad 3 and $W n t / \beta$-catenin pathways promotes vascular smooth muscle cell proliferation. Cell Signal 2016;28:498-505.

12 Thompson MD, Monga SP: WNT/betacatenin signaling in liver health and disease. Hepatology 2007;45:1298-1305.

13 von Toerne C, Schmidt C, Adams J, Kiss E, Bedke J, Porubsky S, et al: Wnt pathway regulation in chronic renal allograft damage. Am J Transplant 2009;9:2223-2239.

14 Joo M, Lee HK, Kang YK: Expression of betacatenin in hepatocellular carcinoma in relation to tumor cell proliferation and cyclin D1 expression. J Korean Med Sci 2003;18:211-217. 
-15 Feng Y, Ren J, Gui Y, Wei W, Shu B, Lu Q, et al: Wnt/ $\beta$-catenin-promoted macrophage alternative activation contributes to kidney fibrosis. J Am Soc Nephrol 2018;29:182-193.

$>16$ Li J, Ren J, Liu X, Jiang L, He W, Yuan $\mathrm{W}$, et al: Rictor/mTORC2 signaling mediates TGF $\beta 1$-induced fibroblast activation and kidney fibrosis. Kidney Int 2015;88:515-527.

-17 Ren J, Li J, Feng Y, Shu B, Gui Y, Wei W, et al: Rictor/mammalian target of rapamycin complex 2 promotes macrophage activation and kidney fibrosis. J Pathol 2017;242:488-499.

$\checkmark 18$ Weischenfeldt J, Porse B: Bone marrowderived macrophages (BMM): isolation and applications. CSH Protoc 2008;2008:pdb. prot5080.

19 Jacques BE, Puligilla C, Weichert RM, FerrerVaquer A, Hadjantonakis AK, Kelley MW, et al: A dual function for canonical Wnt/ $\beta$-catenin signaling in the developing mammalian cochlea. Development 2012;139:4395-4404.

-20 Ma FY, Liu J, Kitching AR, Manthey CL, Nikolic-Paterson DJ: Targeting renal macrophage accumulation via c-fms kinase reduces tubular apoptosis but fails to modify progressive fibrosis in the obstructed rat kidney. Am J Physiol Renal Physiol 2009;296:F177-F185.

-21 MacDonald BT, Tamai K, He X: Wnt/betacatenin signaling: components, mechanisms, and diseases. Dev Cell 2009;17:9-26.

-22 Iglesias DM, Hueber PA, Chu L, Campbell R, Patenaude AM, Dziarmaga AJ, et al: Canonical WNT signaling during kidney development. Am J Physiol Renal Physiol 2007; 293:F494-F500.

23 Gough NR: Focus issue: Wnt and $\beta$-catenin signaling in development and disease. Sci Signal 2012;5:eg2.
4 Nusse R: Wnt signaling in disease and in development. Cell Res 2005;15:28-32.

25 Clevers H, Nusse R: Wnt/ $\beta$-catenin signaling and disease. Cell 2012;149:1192-1205.

26 Lam AP, Flozak AS, Russell S, Wei J, Jain M, Mutlu GM, et al: Nuclear $\beta$-catenin is increased in systemic sclerosis pulmonary fibrosis and promotes lung fibroblast migration and proliferation. Am J Respir Cell Mol Biol 2011;45:915-922.

27 Myung SJ, Yoon JH, Gwak GY, Kim W, Lee $\mathrm{JH}$, Kim KM, et al: Wnt signaling enhances the activation and survival of human hepatic stellate cells. FEBS Lett 2007;581:2954-2958.

28 Wei J, Bhattacharyya S, Tourtellotte WG, Varga J: Fibrosis in systemic sclerosis: emerging concepts and implications for targeted therapy. Autoimmun Rev 2011;10:267-275.

$>29 \mathrm{He}$ BJ, Joiner ML, Singh MV, Luczak ED, Swaminathan PD, Koval OM, et al: Oxidation of CaMKII determines the cardiotoxic effects of aldosterone. Nat Med 2011;17:1610-1618.

30 Dai C, Stolz DB, Kiss LP, Monga SP, Holzman LB, Liu Y: Wnt/beta-catenin signaling promotes podocyte dysfunction and albuminuria. J Am Soc Nephrol 2009;20:1997-2008.

31 He W, Dai C, Li Y, Zeng G, Monga SP, Liu Y: $\mathrm{Wnt} /$ beta-catenin signaling promotes renal interstitial fibrosis. J Am Soc Nephrol 2009; 20:765-776.

32 Edeling M, Ragi G, Huang S, Pavenstädt H, Susztak K: Developmental signalling pathways in renal fibrosis: the roles of Notch, Wnt and Hedgehog. Nat Rev Nephrol 2016;12: 426-439.
33 Woroniecka KI, Park AS, Mohtat D, Thomas DB, Pullman JM, Susztak K: Transcriptome analysis of human diabetic kidney disease. Diabetes 2011;60:2354-2369.

34 Cox SN, Sallustio F, Serino G, Pontrelli P, Verrienti R, Pesce F, et al: Altered modulation of WNT-beta-catenin and PI3K/Akt pathways in IgA nephropathy. Kidney Int 2010;78: 396-407.

35 Kumawat K, Menzen MH, Bos IS, Baarsma $\mathrm{HA}$, Borger P, Roth $\mathrm{M}$, et al: Noncanonical WNT-5A signaling regulates TGF- $\beta$-induced extracellular matrix production by airway smooth muscle cells. FASEB J 2013;27:16311643.

36 Hao S, He W, Li Y, Ding H, Hou Y, Nie J, et al: Targeted inhibition of $\beta$-catenin/CBP signaling ameliorates renal interstitial fibrosis. J Am Soc Nephrol 2011;22:1642-1653.

>37 Madan B, Patel MB, Zhang J, Bunte RM, Rudemiller NP, Griffiths R, et al: Experimental inhibition of porcupine-mediated Wnt O-acylation attenuates kidney fibrosis. Kidney Int 2016;89:1062-1074.

38 Boor P, Ostendorf T, Floege J: Renal fibrosis: novel insights into mechanisms and therapeutic targets. Nat Rev Nephrol 2010;6:643656.

39 Ricardo SD, van Goor H, Eddy AA: Macrophage diversity in renal injury and repair. J Clin Invest 2008;118:3522-3530.

40 Tetsu O, McCormick F: Beta-catenin regulates expression of cyclin D1 in colon carcinoma cells. Nature 1999;398:422-426.

41 Li YJ, Wei ZM, Meng YX, Ji XR: Beta-catenin up-regulates the expression of cyclinD1, c-myc and MMP-7 in human pancreatic cancer: relationships with carcinogenesis and metastasis. World J Gastroenterol 2005;11: 2117-2123 